\title{
On the Semantics of Linking and Importing in Modular Ontologies
}

\author{
Jie Bao ${ }^{1}$, Doina Caragea ${ }^{2}$, and Vasant G Honavar ${ }^{1}$ \\ ${ }^{1}$ Artificial Intelligence Research Laboratory, \\ Department of Computer Science, \\ Iowa State University, Ames, IA 50011-1040, USA \\ \{baojie, honavar\}@cs.iastate.edu \\ 2 Department of Computing and Information Sciences \\ Kansas State University, Manhattan, KS 66506, USA \\ dcaragea@ksu.edu
}

\begin{abstract}
Modular ontology languages, such as Distributed Description Logics (DDL), $\mathcal{E}$-connections and Package-based Description Logics (P-DL) offer two broad classes of approaches to connect multiple ontology modules: the use of mappings or linkings between ontology modules e.g., DDL and $\mathcal{E}$-connections; and the use of importing e.g., P-DL. The major difference between the two approaches is on the usage of "foreign terms" at the syntactic level, and on the local model disjointness at the semantic level. We compare the semantics of linking in DDL and $\mathcal{E}$-connections, and importing in P-DL within the Distributed First Order Logics (DFOL) framework. Our investigation shows that the domain disjointness assumption adopted by the linking approach leads to several semantic difficulties. We explore the possibility of avoiding some of these difficulties using the importing approach to linking ontology modules.
\end{abstract}

\section{Introduction}

Because the web is a network of loosely coupled, distributed, autonomous entities, it is inevitable that the ontologies on the web to be modular, collaboratively built and partially connected. Hence, there is significant interest on modular ontology languages, such as, Distributed Description Logics (DDL) 4], $\mathcal{E}$-connections [129] and Package-based Description Logics (P-DL) [3].

These proposals adopt two broad classes of approaches to asserting semantic relations between multiple ontology modules: the use of mappings or linkings between ontology modules e.g., DDL and $\mathcal{E}$-connections; and the use of importing e.g., P-DL. The major difference between the two approaches has to do with the use of "foreign terms" in ontology modules. In a linked ontology, different modules have disjoint terminologies and disjoint interpretation domains, and semantic relations between ontology modules are only enabled by a set of mapping axioms, such as bridge rules in DDL or $\mathcal{E}$-connections. Therefore, the direct usage of terms defined in one module is forbidden in another module. In contrast, importing allows an ontology module to make direct reference to terms defined in other ontology modules, i.e., importing of foreign terms. 
Serafini et.al. (2005) [15] compare mapping or linking based approaches to the "integration" of multiple ontology modules such as DDL and $\mathcal{E}$-connections by reducing them to the Distributed First Order Logics (DFOL) 6] framework. However, there is little work on the formal investigation of the importing approach to integrating ontology modules. Against this background, we compare the semantics of the two approaches within the DFOL framework, with the study of their strengthes and limitations. Such an investigation reveals that the importing approach, with the removing of the module disjointness assumption adopted by the linking approach, can provide stronger expressivity and avoid many of the semantic difficulties in current modular ontology language proposals.

\section{Desiderata for Modular Ontology Languages}

We first list a set of minimal requirements for modular ontology languages [2] on the semantic web as the basis for our comparison of the semantics of DDL, $\mathcal{E}$-connections and P-DL within the DFOL framework:

1. Localized Semantics. A modular ontology should not only be syntactically modular (e.g. stored in separated XML name spaces), but also semantically modular. That is, the existence of a global model should not be a requirement for integration of ontology modules.

2. Exact Reasoning. The answer to a reasoning problem over a collection of ontology modules should be semantically equivalent to that obtained by reasoning over an ontology resulting from an integration of the relevant ontology modules. Thus, if an ontology $O$ contains $A \sqsubseteq B, B \sqsubseteq C, C \sqsubseteq D$, and a modularized version of $O$ has two modules $M_{1}=\{A \sqsubseteq B\}, M_{2}=\{C \sqsubseteq D\}$ and a semantic connection $r(B, C)$, which represents the modularized version of $B \sqsubseteq C$, the answer to any reasoning problem obtained by integration of $M_{1}, M_{2}$ and $r(B, C)$ should be the same as that obtained by using a sound and complete reasoner on $O$.

3. Directed Semantic Relations. The framework must support directional semantic relations from a source module to a target module. A directional semantic relation affects only the reasoning within the target module and not the source module.

4. Transitive Reusability. Knowledge contained in ontology modules should be directly or indirectly reusable. That is, if a module $A$ reuses module $B$, and module $B$ reuses module $C$, then effectively, module $A$ reuses module C.

Other desiderata that have been considered in the literature include: the ability to cope with local inconsistency or global inconsistency, and local logic completeness. We believe that the desiderata listed above are among the most critical ones for a modular ontology to be semantically sound and practically usable. 


\section{Distributed First Order Logics}

A DFOL knowledge base (KB) [6] (and hence, a DFOL ontology) includes a family of first order languages $\left\{L_{i}\right\}_{i \in I}$, defined over a finite set of indices $I$. We will use $L_{i}$ to refer to the $i$ th module of the ontology. An $(i$-)variable $x$ or ( $i$-)formula $\phi$ occurring in module $L_{i}$ is denoted as $i: x$ or $i: \phi$ (we drop the prefix when there is no confusion).

The semantics of DFOL includes a set of local models and domain relations. For each $L_{i}$, there is an interpretation domain $\Delta_{i}$. Let $M_{i}$ be the set of all first order models of $L_{i}$ on $\Delta_{i}$. We call each $m \in M_{i}$ a local model of $L_{i}$. A domain relation $r_{i j}$, where $i \neq j$, is a subset of $\Delta_{i} \times \Delta_{j}$. The domain relation $r_{i j}$ represents the capability of the module $j$ to map the objects of $\Delta_{i}$ in $\Delta_{j}$, or, the $j$ 's subjective view of the relation between $\Delta_{i}$ and $\Delta_{j}$. In general, $r_{i j} \neq r_{j i}^{-}$.

We use $\left\langle d, d^{\prime}\right\rangle$ in $r_{i j}$ to denote that from the point of view of $j$, the object $d$ in $\Delta_{i}$ is mapped to the object $d^{\prime}$ in $\Delta_{j} ; d$ is called a pre-image of $d^{\prime}$, and $d^{\prime}$ is called an image of $d$. In general, domain relations can be injective, surjective, bijective, or arbitrary. For an object $d \in \Delta_{i}, r_{i j}(d)$ denotes the set $\left\{d^{\prime} \in \Delta_{j} \mid\left\langle d, d^{\prime}\right\rangle \in r_{i j}\right\}$. For a subset $D \subseteq \Delta_{i}, r_{i j}(D)$ denotes $\cup_{d \in D} r_{i j}(d)$, is the image set of $D$.

\section{Semantics of Linking in DDL}

One influential family of modular ontology formalisms is the linking approach. The linking approach is aimed at preserving the autonomy of loosely coupled modules, while allowing restricted "mappings" between formulae of linked modules. Formally, a linking approach holds the follow assumptions:

- For any $L_{i}$ and $L_{j}, i \neq j, i$-terms and $j$-terms are disjoint.

- The semantic connection between $L_{i}$ and $L_{j}$ is enabled only by mapping rules between $i$-terms and $j$-terms, which are interpreted as domain relations $r_{i j} \subseteq \Delta_{i} \times \Delta_{j}$.

- Local interpretation domains and domain relations are disjoint. For any $i \neq$ $j, \Delta_{i} \times \Delta_{i}$ (or $\Delta_{j} \times \Delta_{j}$ ) has intersection neither with $r_{i j}$ nor with $r_{j i}$.

Based on DFOL, Distributed Description Logics (DDL) 4 is one of the first linking-based modular ontology formalisms. In DDL, the semantic mappings between disjoint modules $L_{i}$ and $L_{j}$ are established by a set of inter-module axioms called "Bridge Rules" $\left(B_{i j}\right)$ of the form:

- INTO rule: $i: \phi \stackrel{\sqsubseteq}{\rightrightarrows} j: \psi$, semantics: $r_{i j}\left(\phi^{m_{i}}\right) \subseteq \psi^{m_{j}}$

- ONTO rule: $i: \phi \stackrel{\sqsupseteq}{\longrightarrow} j: \psi$, semantics: $r_{i j}\left(\phi^{m_{i}}\right) \supseteq \psi^{m_{j}}$

where $m_{i}\left(m_{j}\right)$ is a model of $L_{i}\left(L_{j}\right), \phi, \psi$ are formulae, $r_{i j}$ is a domain relation which serves as the interpretation of $B_{i j}$. Note that $B_{i j}$ is directional. We will only consider bridge rules between concepts, not roles [5], since there is still no reasoning support for role bridge rules 14. 
Distributed concept correspondence between two modules in DDL covers some important scenarios that require mapping between ontology modules. However, the expressivity of DDL is limited in some settings that arise in practical applications: For example, DDL cannot be used to express "a person $x$ works in a region $y$ ". In general, it can not construct new concepts using terms across modules, such as restrictions $\forall 1: R .2: D$ and $\exists 1: R .2: D$, where $C, D$ are concepts and $R$ is role.

In addition to the expressivity limitations, DDL may present semantic difficulties in some situations. While DDL bridge rules are intended to simulate concept inclusions 45], arbitrary modelling with bridge rules may lead to undesired semantics, such as in the Subsumption Propagation problem and Inter-module Unsatisfiability problem, as noted in 977:

Example 1 (Subsumption Propagation). A KB $\Sigma_{d}$ includes modules $L_{\{1,2,3\}}$, each with an empty TBox; bridge rules $B_{12}=\{1:$ Bird $\stackrel{\sqsupseteq}{\longrightarrow} 2:$ Fowl $\}, B_{23}=\{2$ : Fowl $\stackrel{\sqsupseteq}{\rightrightarrows} 3$ : Chicken $\}$. The entailment problem $1:$ Bird $\stackrel{\sqsupseteq}{\longrightarrow}$ : Chicken cannot be answered since bridge rules $B_{13}$ are not given, nor can be inferred.

Note that bridge rules may be inferred between the same pair of modules. For example, if $1: A \stackrel{\sqsubseteq}{\rightrightarrows} 2: B$ and $2: B \sqsubseteq 2: C$, it can be inferred that $1: A \stackrel{\sqsubseteq}{\leftrightarrows} 2$ : $C$. Intra-module subsumption may also be reused in some particular cases. For example, if $1: A \sqsubseteq 1: B, 1: A \stackrel{\sqsupseteq}{\rightrightarrows} 2: C$ and $1: B \stackrel{\sqsubseteq}{\rightrightarrows} 2: D$, it can be inferred that $2: C \sqsubseteq 2: D[16$. However, Example 1 shows that in general bridge rules in DDLs are not transitively reusable, thereby are restricted for many application scenarios.

Example 2 (Inter-module Unsatisfiability[9]7]). DDLs may not detect unsatisfiability across ontology modules. A KB $\Sigma_{d}$ includes modules $L_{\{1,2\}}, L_{1}=$ $\{1:$ Bird $\sqsubseteq 1:$ Fly $\}, L_{2}=\{2:$ Penguin $\sqsubseteq \top\}, B_{12}=\{1:$ Bird $\sqsupseteq 2$ : Penguin, $1: \neg$ Fly $\stackrel{\sqsupseteq}{\rightrightarrows} 2$ : Penguin $\}$. Penguin is still satisfiable in $\Sigma_{d}$.

Such difficulties are rooted in the implicit local domain disjointness assumption of DDL: individuals in each local domain are private to that domain, and DDL semantics does not take into account if individuals in different local domains may represent the same physical world object. Therefore, a bridge rule, while intended to simulate concept inclusion, cannot be read directly as concept inclusion, such as $i: A \sqsubseteq j: B$. Instead, it must be read as a classic DL axiom in the following way 4:

$$
\begin{aligned}
& -i: A \stackrel{\sqsubseteq}{\rightrightarrows} j: B \Rightarrow(i: A) \sqsubseteq \forall R_{i j} \cdot(j: B) \\
& -i: A \stackrel{\sqsupseteq}{\longrightarrow} j: B \Rightarrow(j: B) \sqsubseteq \exists R_{i j}^{-} \cdot(i: A)
\end{aligned}
$$

where $R_{i j}$ is a new role representing correspondences $B_{i j}$ between $L_{i}$ and $L_{j}$. Such translations are best understood as shown in Figure 1.

Therefore, for the given subsumption propagation example, if $B_{13}=\varnothing$, entailment Chicken $\sqsubseteq \exists R_{13}^{-}$.Bird is not always true. For the inter-module unsatisfiability problem, concept Penguin $\left(\sqsubseteq \exists R_{12}^{-} \cdot(F l y) \sqcap \exists R_{12}^{-} \cdot(\neg F l y)\right.$ ) is satisfiable. 


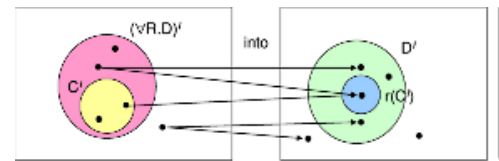

(a) Into Bridge Rules

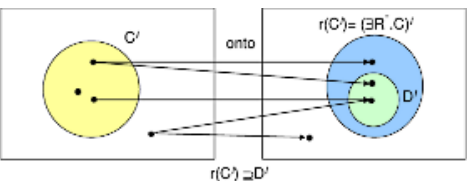

(b) Onto Bridge Rules

Fig. 1. Semantics of DDL Bridge Rules

Thus, the semantics of DDL are designed to simulate concept inclusion with a special type of roles, i.e., bridge rules. However, in the absence of a principled approach to avoid arbitrary domain relation interpretations for bridge rules, all semantic relations (bridge rules) between DDL modules are localized to pairs of modules that are bridged by the rules in question. Consequently, semantic relations between a pair of DDL modules cannot be safely reused by other modules, thereby precluding general subsumption propagation, and more generally, module transitive reusability. Note further that in order to enable distributed (not necessarily exact) reasoning in general, a DDL KB needs explicit declaration of domain relations between each pair of modules, leading to an exponential blowup in the number of bridge rules, with the attendant inefficiency and increased risk of inconsistencies.

Serafini et al. 14] has asserted that the inter-module unsatisfiability difficulty is the result of incomplete modelling. They have argued that it can be eliminated if extra information, for example, $1: \neg$ Bird $\stackrel{\sqsubseteq}{\rightrightarrows} 2: \neg$ Penguin and $1:$ Fly $\stackrel{\sqsubseteq}{\longrightarrow} \neg 2$ : Penguin, is added to guarantee one-to-one domain relations. Our investigation reveals a more general result: one-to-one domain relations can guarantee that reasoning over DDL always yields the same result as that obtained from an integrated ontology when bridge rules are replaced with general concept inclusions (GCI). First, we have the definition:

Definition 1. A domain relation $r_{i j}$ for bridge rules $B_{i j}$ is said to be one-toone if for any bridge rule $C \stackrel{\sqsubseteq}{\rightrightarrows} D$ or $C \stackrel{\sqsupseteq}{\rightrightarrows} D$, for any $x \in C^{\mathcal{I}_{i}}$, there is one and only one unique $y \in \Delta_{j}$ such that $\langle x, y\rangle \in r_{i j}$.

The integration process from a DDL ontology to a ordinary (global) DL ontology is given in [4. For a DDL ontology $\left\{L_{i}\right\}$, the global DL (GDL) ontology is defined as follows:

- There is a new top concept $\top_{g}$ and a new bottom concept $\perp_{g}$ in GDL.

- The primitive concepts of GDL consist of $i: A$ obtained from primitive concepts or constant concepts $A$ (such as $\top_{i}$ and $\perp_{i}$ ) of $L_{i}$

- The primitive roles of GDL include $i: p$ obtained from primitive or constant roles $p$ of $L_{i}$

The mapping \#() from concepts/roles in $L_{i}$ to concepts/roles in GDL is defined as follows: for atomic concepts, roles, and individuals $i: M, \#(i: M)=i: M$; 
for a complex concept constructor $\rho$ with $k$ arguments, $\#\left(i: \rho\left(X_{1}, \ldots, X_{k}\right)\right)=$ $\top_{i} \sqcap \rho\left(\#\left(X_{1}\right), \ldots, \#\left(X_{k}\right)\right)$. For example, $\# i:(\forall p . C)=\top_{i} \sqcap \forall(i: p) \cdot\left(\top_{i} \sqcap i: C\right)$.

Applying \#() to a DDL knowledge base $\Sigma=\left\langle\left\{L_{i}\right\},\left\{B_{i j}\right\}\right\rangle$, we get an integrated GDL [4] \#( $\Sigma)$ that contains:

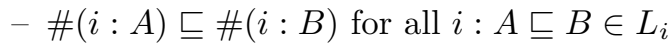

$-\perp_{i} \sqsubseteq \perp_{g}$

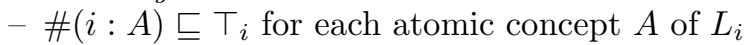

- Axioms that ensure the domain and range of any $i$-role to be $\top_{i}$ : $\top_{i} \sqsubseteq \forall(i$ : $s) . \top_{i}, \neg \top_{i} \sqsubseteq \forall(i: s) \cdot \perp_{g}$

However, in contrast to the approach taken in 4, we will translate bridge rules in DDL as GCIs in GDL. Hence, $\#(\Sigma)$ will include in addition to the above:

- \#(i:C)巨\#(j:D) for all $i: C \stackrel{\sqsubseteq}{\longrightarrow} j: D \in B_{i j}$

- \# $(i: C) \sqsupseteq \#(j: D)$ for all $i: C \stackrel{\sqsupseteq}{\longrightarrow} j: D \in B_{i j}$

Since the motivation of DDL bridge rules is to simulate concept subsumption as mentioned in DDL proposals [4514, we believe that GCIs offer a more appropriate translation for bridge rules in comparing the result of reasoning in the distributed setting with that of the centralized setting. Note that the semantic difficulties of DDL under incomplete modelling is actually due to the semantic differences between concept subsumptions (i.e., GCIs) and bridge rules (as shown in the Examples 1 and 2). The following theorem reveals that the domain relations being one-to-one is a sufficient condition for exact reasoning in DDL if bridge rules are intended to represent inter-module concept inclusions (proof can be found in the longer version of the paper 1 ).

Theorem 1. Suppose $\Sigma=\left\langle\left\{L_{i}\right\},\left\{B_{i j}\right\}\right\rangle$ is a DDL KB, where none of $L_{i}$ uses role constants or role constructors, and all domain relations in all models of $\Sigma$ are one-to-one, then

$$
\begin{aligned}
& \text { - \#( }) \vDash \#(i: X) \sqsubseteq \#(i: Y) \text { if and only if } \Sigma \vDash_{d} i: X \sqsubseteq i: Y \\
& -\#(\Sigma) \vDash \#(i: X) \sqsubseteq \#(j: Y) \text { if and only if } \Sigma \vDash_{d}(i: X \stackrel{\sqsubseteq}{\longrightarrow} j: Y \text { or } \\
& \quad(j: Y \stackrel{\sqsupseteq}{\rightrightarrows} i: X)
\end{aligned}
$$

At present, there is no principled approach in DDL to specify such domain relations. Adding $\neg C \stackrel{\sqsubseteq}{\longrightarrow} \neg D$ for each $C \stackrel{\sqsupseteq}{\longrightarrow} D$, as suggested in [14], does not necessarily result in injective (and hence, also not one-to-one) domain relations for any inter-module concept relations.

Example 3. $A K B \Sigma_{d}$ includes modules $L_{\{1,2\}}$, TBox of $L_{1}$ is $\{$ Woman $\equiv$ $\neg$ Man\}, TBox of $L_{2}$ is $\{$ Girl $\equiv \neg$ Boy $\}$; bridge rules $B_{12}=\{1:$ Man $\sqsupseteq$ 2 : Boy\}. According to [14, we should also add $\neg 1:$ Man $\stackrel{\sqsubseteq}{\rightrightarrows} \neg$ : Boy i.e.

\footnotetext{
${ }^{1}$ http://archives.cs.iastate.edu/documents/disk0/00/00/04/08/index.html
} 
$1:$ Woman $\stackrel{\sqsubseteq}{\rightrightarrows} 2:$ Girl to $B_{12}$. However, that doesn't rule out the possibility of a Girl object being both an image of a Man object and a Woman object, neither ensure one-to-one correspondence between Man objects and Boy objects.

Example 4. (adopted from [17]) Module $L_{1}$ entails $\top \sqsubseteq 1$ : Car, module $L_{2}$ entails UsefulThing $\sqsubseteq \neg$ UselessThing, and there are bridge rules 1 : Car $\leftrightarrows$ 2 : UsefulThing and $1:$ Car $\stackrel{\sqsubseteq}{\longrightarrow} 2$ :UselessThing. There is no required new bridge rules to be added according to [14. However, 1 : Car is not unsatisfiable, since DDL semantics allows empty domain relations.

DDL, as presented in 4, meets the localized semantics and directional semantic relations requirements, but not the exact reasoning and transitive reusability requirements. In general, DDL in its present form does not provide a satisfactory formalism for inter-module or inter-ontology subsumption. In the following text, we will show it can be improved by restricting domain relations to be one-to-one, by P-DL or a combination of DDL and $\mathcal{E}$-connections.

\section{Semantics of Linking in $\mathcal{E}$-Connections}

While DDL allows only one type of domain relations, $\mathcal{E}$-connections allow multiple "link" relations between two domains, such as worksIn and bornIn between 2 : Person and 1 : Region. $\mathcal{E}$-connections between ADSs [12], and in particular, between DLs 119, restrict the local domains of the $\mathcal{E}$-connected ontology modules to be disjoint. Roles are divided into disjoint sets of local roles (connecting concepts in one module) and links (connecting inter-module concepts).

Formally, given ontology modules $\left\{L_{i}\right\}$, an (one-way binary) link (more expressive $\mathcal{E}$-connections are beyond the scope of this paper) $E \in \mathcal{E}_{i j}$, where $\mathcal{E}_{i j}, i \neq j$ is the set of all links from the module $i$ to the module $j$, can be used to construct a concept in module $i$, with the syntax and semantics specified as follows:

- $\langle E\rangle(j: C)$ or $\exists E .(j: C):\left\{x \in \Delta_{i} \mid \exists y \in \Delta_{j},(x, y) \in E^{M}, y \in C^{M}\right\}$

- $\left.\forall E .(j: C):\left\{x \in \Delta_{i} \mid \forall y \in \Delta_{j},(x, y) \in E^{M} \rightarrow y \in C^{M}\right\}\right\}$

where $M=\left\langle\left\{m_{i}\right\},\left\{E^{M}\right\}_{E \in \mathcal{E}_{i j}}\right\rangle$ is a model of the $\mathcal{E}$-connected ontology, $m_{i}$ is the local model of $L_{i} ; C$ is a concept in $L_{j}$, with interpretation $C^{M}=C^{m_{j}}$; $E^{M} \subseteq \Delta_{i} \times \Delta_{j}$ is the interpretation of an $\mathcal{E}$-connection $E$. $\mathcal{E}$-connections also permit number restrictions on links [12.

An $\mathcal{E}$-connection model $M$ can be mapped to a DFOL model $M_{d}=\left\langle\left\{m_{i}\right\},\left\{r_{i j}\right\}\right\rangle$ with each $E^{M}\left(E \in \mathcal{E}_{i j}\right)$ acting as a domain relation $r_{i j}[15$. Extending the semantics of $\mathcal{E}$-connection axioms $((1)$ and $(3)$ below) given in 15 so as to allow the use of constructed concepts $(\exists E . D$ and $\forall E . D)$ on either side of the subsumption, we have (also see Figure 2):

1) $C \sqsubseteq \forall E . D: E^{M}\left(C^{m_{i}}\right) \subseteq D^{m_{j}}$

2) $C \sqsupseteq \forall E . D:(\neg C)^{m_{i}} \subseteq\left(E^{M}\right)^{-}\left((\neg D)^{m_{j}}\right)$, i.e., $\forall x \in \Delta_{i}, E^{M}(x) \subseteq D^{m_{j}} \rightarrow$ $x \in C^{m_{i}}$ 


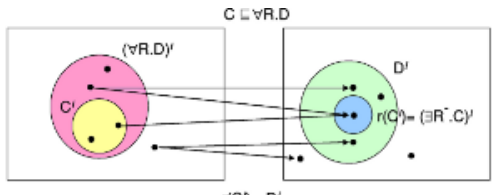

(a) $C \stackrel{\text { (C) }}{\sqsubseteq} \forall E . D$

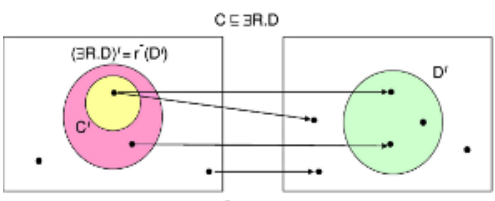

(c) $C \stackrel{i(0)}{\sqsubseteq}=\stackrel{C^{\prime}}{\exists} E . D$

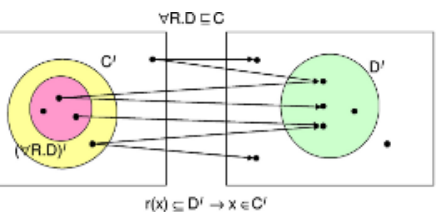

(b) $C \sqsupseteq \forall E . D$

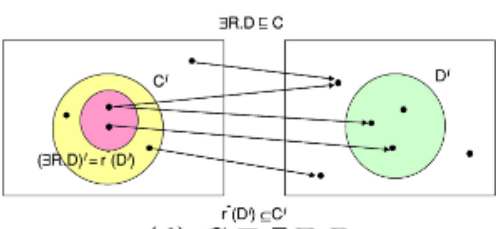

(d) $C \sqsupseteq \exists E . D$

$r$ is the interpretation of link $E$, also acting as the domain relation between the two local domain.

Fig. 2. Semantics of $\mathcal{E}$-Connections

3) $C \sqsubseteq \exists E . D: C^{m_{i}} \subseteq\left(E^{M}\right)^{-}\left(D^{m_{j}}\right)$

4) $C \sqsupseteq \exists E . D: E^{M}\left((\neg C)^{m_{i}}\right) \subseteq(\neg D)^{m_{j}}$, i.e., $C^{m_{i}} \supseteq\left(E^{M}\right)^{-}\left(D^{m_{j}}\right)$

where $\left(E^{M}\right)^{-}$is the inverse of $E^{M}, C$ is an $i$-concept and $D$ is a $j$-concept, $C$ can be an atomic or complex concept. Note that case (2)(similarly also for (4)) can not be reduce to defining $C^{\prime} \equiv \forall E . D$ and $C^{\prime} \sqsubseteq C$ in $i$, since $\equiv$ is the short for $\sqsubseteq$ and $\sqsupseteq$.

It has been argued that $\mathcal{E}$-connections are more expressive than DDL 127 because DDL can be reduced to $\mathcal{E}$-connections. However, the reliance of the reduction on the equivalence of $C \stackrel{\sqsubseteq}{\longrightarrow} D$ to $\langle E\rangle . C \sqsubseteq D$ and $C \stackrel{\sqsupseteq}{\longrightarrow} D$ to $\langle E\rangle . C \sqsupseteq D$ [127], presents semantic difficulties with regard to DDL and $\mathcal{E}$ connections semantics in the DFOL framework [15]: ONTO $(\stackrel{\sqsupseteq}{\rightrightarrows})$ rules in DDL is actually mapped to type $d$ interpretation constraints in DFOL while $\langle E\rangle . C \sqsupseteq D$ is mapped to type $b$ interpretation constraints in DFOL.

We show that inverse links being allowed is a necessary condition for $\mathcal{E}$ connections to be more expressive than DDL bridge rules:

Theorem 2. $\mathcal{E}$-connections, as presented in [127] is strictly more expressive than DDL as presented in [4], only if inverse links are allowed.

Proof Sketch: Comparison of the semantics of DDL and $\mathcal{E}$-connections, if we treat the only domain relation in DDL as a $\mathcal{E}$-connection $E$, as shown in [154], $C \sqsubseteq \forall E . D$ has the same semantics as the "into" rule $C \stackrel{\sqsubseteq}{\longrightarrow} D\left(r_{i j}\left(C^{m_{i}}\right) \subseteq D^{m_{j}}\right)$. However, onto rules, such as $C \stackrel{\sqsupseteq}{\longrightarrow} D\left(r_{i j}\left(C^{m_{i}}\right) \supseteq D^{m_{j}}\right)$, can be translated into $D \sqsubseteq \exists E^{-} . C$ only if the inversion of $\mathcal{E}$-connections is allowed.

Thus, the language $\mathcal{C}_{\mathcal{H}}^{\mathcal{E}}(\mathcal{S H I} \mathcal{H}, \mathcal{S H O} \mathcal{Q}, \mathcal{S H \mathcal { O }})$ is more expressive than DDL but $\mathcal{C}_{\mathcal{H} \mathcal{Q}}(\mathcal{S H \mathcal { I }}, \mathcal{S H O} \mathcal{Q}, \mathcal{S H \mathcal { O }})$ (allowing no inverse link) [7] is not. 
Note that for $i: C \stackrel{\sqsupseteq}{\longrightarrow} j: D$, defining an $\mathcal{E}$-connection $F$ from $j$ to $i$, the onto rule still cannot be translated into $D \sqsubseteq \exists F$. $C$, since DDL semantics doesn't assume $r_{i j}=r_{j i}^{-}$, therefore $F \neq E^{-}$. To assert the inverse relation, we still need inverse link constructors in $\mathcal{E}$-connections.

$\mathcal{E}$-connections allow multiple links between modules and the construction of new concepts e.g. WorkForce $\equiv\langle$ worksIn $\rangle$ Region, while DDL does not. Module transitive useability can be realized in a limited form by transitive links [13]. $\mathcal{E}$-connections are also directional. Reasoning in $\mathcal{E}$-connections without generalized links is exact w.r.t a combined TBox of the $\mathcal{E}$-connected ontology, since a concept is satisfiable in the $\mathcal{E}$-connected ontology if and only if there is a combined model for the combined TBox and the concept 98 .

However, the applicability of $\mathcal{E}$-connections in practice is also limited by the need to ensure that the local domains are disjoint:

- To enforce local domain disjointness, a concept cannot be declared as subclass of another concept in a foreign module thereby ruling out the possibility of asserting inter-module subsumption and the general support for transitive useability; a property cannot be declared as sub-relation of a foreign property; neither foreign classes nor foreign properties can be instantiated; cross-module concept conjunction or disjunction are also illegal.

- $\mathcal{E}$-connected ontologies have difficulties to be used with OWL importing mechanism, since importing may actually "decouple" the combination and result in inconsistency [7].

\section{Semantics of Importing - P-DL}

Our investigation of the semantics of DDL and $\mathcal{E}$-connections suggests that many of the semantic difficulties of linking approaches might be the result of a fundamental assumption that the local language and local models are disjoint. Thus, it is interesting to consider formalisms that relax this assumption.

OWL does not make such module disjointness assumption. Instead, it adopts an importing mechanism to support integration of ontology modules. However, the importing mechanism in OWL, in its current form, suffers from several serious drawbacks: (a) It directly introduces both terms and axioms of the imported ontologies into the importing ontology, and thus fails to support local semantics (b) It provides no support for partial reuse of an ontology module.

Package-based Description Logics (P-DL) 3 offer a tradeoff between the strong module disjointness assumption of DDL and $\mathcal{E}$-connections, and the $\mathrm{OWL}$ importing mechanics, which forces complete overlapping of modules.

\subsection{Syntax and Semantics of P-DL}

In P-DL, an ontology is composed of a collection of modules called packages. Each term (name of a concept, a property or an individual) and each axiom is associated with a home package. A package can use terms defined in other packages i.e., foreign terms. If a package $L_{j}$ uses a term $i: t$ with home package 
$L_{i}(i \neq j)$, then we say $t$ is imported into $L_{j}$, and the importing relation is denoted as $r_{i j}^{t}$. $L_{i}$ may contain the usual TBox and ABox of DL. For simplicity, we do not present advanced features of P-DL, such as package hierarchy and scope limitation modifiers [3].

We denote the package extension to DL as $\mathcal{P}$. For example, $\mathcal{A} \mathcal{L C} \mathcal{P}$ is the package-based version of $\mathrm{DL} \mathcal{A} \mathcal{L C}$. In what follows, we will examine a restricted type of package extension which only allows import of concept names, denoted as $\mathcal{P}_{\mathcal{C}}$. We will show that even this restricted form of package extension is not trivial and is more expressive than DDL and $\mathcal{E}$-connection.

The semantics of P-DL is expressed in DFOL as follows: For a package-based ontology $\left\langle\left\{L_{i}\right\},\left\{r_{i j}^{t}\right\}_{i \neq j}\right\rangle$, a distributed model is $M=\left\langle\left\{m_{i}\right\},\left\{\left(r_{i j}^{t}\right)^{M}\right\}_{i \neq j}\right\rangle$, where $m_{i}$ is the local model of module $i,\left(r_{i j}^{t}\right)^{M} \subseteq \Delta_{i} \times \Delta_{j}$ is the interpretation for the importing relation $r_{i j}^{t}$, which meets the following requirements:

- Every importing relation is one-to-one, and for every object in $t^{m_{j}}$ there is a single unique object in $t^{m_{i}}$ as its pre-image (therefore $r_{i j}\left(t^{m_{i}}\right)=t^{m_{j}}$ ).

- Term Consistency: importing relations are consistent for different terms. Each object in the model of a source package corresponds uniquely to an object in the model of any target package for interpretations of importing relations of all terms, i.e., for any $i: t_{1} \neq i: t_{2}$ and any $x, x_{1}, x_{2} \in \Delta_{i}$, $\left(r_{i j}^{t_{1}}\right)^{M}(x)=\left(r_{i j}^{t_{2}}\right)^{M}(x)$ and $\left(r_{i j}^{t_{1}}\right)^{M}\left(x_{1}\right)=\left(r_{i j}^{t_{2}}\right)^{M}\left(x_{2}\right) \neq \varnothing \rightarrow x_{1}=x_{2}$.

- Compositional Consistency: if $\left(r_{i k}^{i: t_{1}}\right)^{M}(x)=y_{1},\left(r_{i j}^{i: t_{2}}\right)^{M}(x)=y_{2},\left(r_{j k}^{j: t_{3}}\right)^{M}$ $\left(y_{2}\right)=y_{3}$, , (where $t_{1}$ and $t_{2}$ may or may not be same), and $y_{1}, y_{2}, y_{3}$ are not null, then $y_{1}=y_{3}$. Compositional consistency helps ensure that the transitive reusability property holds for P-DL.

The domain relation between $m_{i}$ and $m_{j}$ is $r_{i j}=\cup_{t}\left(r_{i j}^{t}\right)^{M}$.

Lemma 1. Domain relations in a P-DL model are one-to-one.

Lemma 1 states that a domain relation $r_{i j}$ in a P-DL model isomorphically maps, or copies, the relevant partial domain from $m_{i}$ to $m_{j}$. For any concept $i: C, r_{i j}\left(C^{m_{i}}\right)$, if not empty, contains the copy of a subset of objects in $C^{m_{i}}$. Such domain relations allow us to relax the domain disjointness assumption adopted in DDL and $\mathcal{E}$-connections, since the construction of a local model is partially dependent on the structure of local models of imported modules, with the benefits of preserving exact semantics of terms shared by different modules.

Immediately from the one-to-one domain relation property, we have:

Lemma 2. In a P-DL model $m$, for any domain relation $r_{i j}$ and concept $i: C$, we have $r_{i j}\left(C^{m_{i}}\right) \cap r_{i j}\left((\neg C)^{m_{i}}\right)=\varnothing$.

If a term is indirectly used in a non-home package, compositional consistency property of domain relations makes the domain relation inferrable. For example, if some terms defined in $L_{1}$ are imported into $L_{2}$, and some terms in $L_{2}$ are imported into $L_{3}$, then the importing relation $r_{13}$ can be inferred from the composition $r_{12} \circ r_{23}$. 
Lemma 3. For domain relations in a model of $P$-DL, $r_{i k}=r_{i j} \circ r_{j k}, i \neq j, j \neq k$.

In the following text, $r_{i j}$ refers to either an explicitly given domain relation or an inferred domain relation, or their union, between package $i$ and $j$.

If $i: C$ is imported into $j$, we define inter-module subsumption $i: C \bigsqcup_{P} j: D$ as $r_{i j}\left(C^{m_{i}}\right)=C^{m_{j}} \subseteq D^{m_{j}}$ and $i: C \sqsupseteq_{P} j: D$ as $r_{i j}\left(C^{m_{i}}\right)=C^{m_{j}} \supseteq D^{m_{j}}$ (see Figure 3). Note that inter-module subsumption is substantially different from bridge rules in DDL. DDL bridge rules bridge semantic gaps between different concepts, and there is no principled way to ensure subjective domain relations to be semantically consistent (in the sense of one-to-one mappings and compositional consistency). In contrast, P-DL importing mechanism bridges the semantic gaps between multiple references of the same concept in different modules. Importing of $C$ from $i$ to $j$ cannot be reduced to a DDL equivalency bridge rule $C \stackrel{\equiv}{\longrightarrow} C^{\prime}$, since in DDL $r_{i j}\left(C^{m_{i}}\right)=C^{\prime m_{j}}$ does not guarantee $C^{m_{i}}$ and $C^{\prime m_{j}}$ are interpretations for the same concept.

We show below that such a relaxation of module disjointness does not sacrifice localized semantics and can help us to solve many semantic difficulties presented in other approaches and provide stronger expressivity.

\subsection{Features of P-DL Semantics}

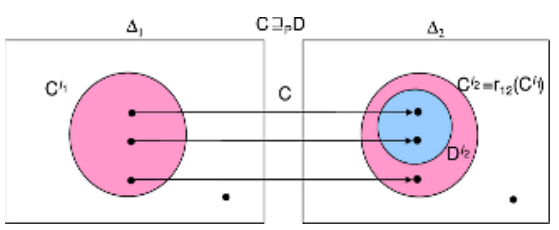

Fig. 3. Semantics of P-DL

The loss of local model disjointness in P-DL does not sacrifice localized semantics property of modules, since the local models (unlike in OWL which requires completely overlapping of local models) are, only partially overlapping. The semantics of the part of a module that is not exported to any other module remains local to that module. Consequently, there is no required global model. The example below demonstrates that P-DL also satisfies directional semantic relation and module transitive reusability properties.

Example 5. Consider four modules $L_{\{1,2,3,4\}}$ as shown in Figure 4.

1. Transitivity of inter-module subsumption holds: $r_{14}\left(A^{m_{1}}\right)=r_{24}\left(r_{12}\left(A^{m_{1}}\right)\right) \subseteq$ $r_{24}\left(r_{12}\left(B^{m_{1}}\right)\right)=r_{24}\left(B^{m_{2}}\right) \subseteq r_{24}\left(C^{m_{2}}\right) \subseteq r_{24}\left(P^{m_{2}}\right)=P^{m_{4}} \subseteq Q^{m_{4}}$, i.e., $A \sqsubseteq_{P} Q$. Although no term in $L_{1}$ is directly imported into $L_{4}$, we can infer the domain relation $r_{14}$ from $r_{12 j} \circ r_{24}$ utilizing their compositional consistency property.

2. The importing relation is directional. Thus, $r_{12}\left(A^{m_{1}}\right) \subseteq r_{12}\left(D^{m_{1}}\right)$ is enforced only in $L_{2}$, while $A^{m_{1}} \subseteq D^{m_{1}}$ is not required in $L_{1}$. There is no information "backflow" in importing. Therefore, while $L_{2}$ and $L_{3}$ are inconsistent, they are all consistent to $L_{1}$, and the consistency of $L_{1}$ is still guaranteed.

3. The model overlapping is only partial, e.g., E and $F$ in 1 are semantically separated from $L_{2}$ and have no correspondence in the local model $m_{2}$. 


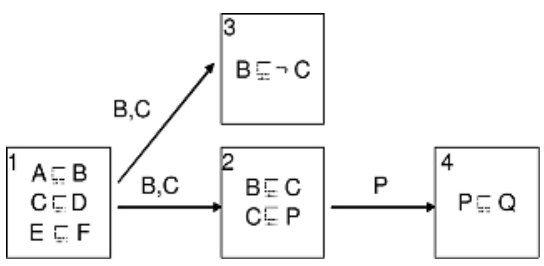

Fig. 4. P-DL Ontology Example

An integrated $\mathrm{KB}$ can be obtained from a P-DL KB by combining axioms in all packages. Because of the isomorphic nature of importing relations, we have the theorem (proof is in the longer version of the paper):

Theorem 3. Reasoning in a $P-D L K B$ is exact w.r.t. its integrated $K B$.

The intuition behind this proof is as follows: since the only type of semantic relations between modules are importing relations, and shared terms are always interpreted consistently in different modules, we can transform a distributed PDL model into a classic DL model by merging all "copied" objects in each of the local models.

However, a limitation of the importing approach adopted by P-DL is that the general decidability transfer property does not always hold in P-DL since the union of two decidable fragments of DL may not in general be decidable [1. This presents semantic difficulties in the general setting of connecting ADSs 1112. Fortunately, in the case of a web ontology language where different ontology modules are specified using subsets of the same decidable DL language such as $\mathcal{S H O I Q}(D)$ (OWL-DL), the union of such modules is decidable. With the availability of the decision procedure [10] and highly optimized reasoners for $\mathcal{S H O I Q}(D)$, we can ensure the decidability of P-DL ontology within the modular web ontology setting. Therefore we have:

\section{Theorem 4. $\mathcal{S H O I} \mathcal{Q P}_{\mathcal{C}}(D)$ is decidable}

P-DL also has some expressivity limitations. $\mathcal{P}_{\mathcal{C}}$ does not allow role inclusions across different modules, using foreign role to construct local concept, declaring a local role as the inverse of a foreign role, nor the importing of nominals defined in other modules. Due to the one-to-one domain relation requirement, $\mathrm{P}$-DL can support only one-to-one ABox mappings, and not many-to-one, one-to-many, or many-to-many ABox mappings. The semantics of more expressive P-DL that allows importing of role and individual names still needs further investigation.

\subsection{Relation Between P-DL, DDL and $\mathcal{E}$-Connections}

P-DL, despite its stronger domain relation restrictions, can be used to model DDLs and $\mathcal{E}$-Connections.

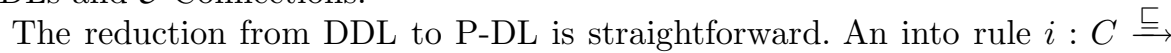
$j: D$ in DDL can be reduced to a P-DL axiom $C \sqsubseteq_{P} D$ in module $j$ and $C$ is an imported concept; similarly, an onto rule $i: C \stackrel{\sqsupseteq}{\longrightarrow} j: D$ in DDL is translated as $C \sqsupseteq_{P} D$ in module $j$ and $C$ is an imported concept. The semantic interpretation of such a reduction is clear since $r_{i j}\left(C^{m_{i}}\right)=C^{m_{j}}$, therefore $r_{i j}\left(C^{m_{i}}\right) \subseteq D^{m_{j}}$ iff $C^{m_{j}} \subseteq D^{m_{j}}$ and $r_{i j}\left(C^{m_{i}}\right) \supseteq D^{m_{j}}$ iff $C^{m_{j}} \supseteq D^{m_{j}}$.

P-DL may avoid the semantic difficulties presented in DDL. 
Example 6 (Subsumption Propagation). A P-DL KB includes three modules $L_{\{1,2,3\}}, L_{1}$ has empty TBox, $L_{2}=\{1:$ Bird $\sqsupseteq 2:$ Fowl $\}, L_{3}=\{2$ : Fowl $\sqsupseteq 3$ : Chicken $\}$, importing relations are $r_{12}^{\text {Bird }}, r_{23}^{\text {Fowl }}$. The inter-module subsumption problem $1:$ Bird $\sqsupseteq 3:$ Chicken can be answered, since in any model of the KB, Chicken ${ }^{m_{3}} \subseteq r_{23}\left(\right.$ Fowl $\left.^{m_{2}}\right) \subseteq r_{23}\left(r_{12}\left(\right.\right.$ Bird $\left.\left.^{m_{1}}\right)\right)=r_{13}\left(\right.$ Bird $\left.^{m_{1}}\right)$, therefore Bird $\sqsupseteq_{P}$ Chicken is always true.

Example 7 (Inter-module Unsatisfiability). A $P$-DL ontology contains two modules $L_{\{1,2\}}, L_{1}=\{1:$ Bird $\sqsubseteq 1:$ Fly $\}, L_{2}=\{1:$ Bird $\sqsupseteq 2:$ Penguin, $\neg 1$ : Fly $\sqsupseteq 2:$ Penguin $\}$, importing relations are $r_{12}^{\text {Bird }}, r_{12}^{\text {Fly }}$. For any model $m$ of the ontology, Penguin ${ }^{m_{2}} \subseteq r_{12}\left((\neg F l y)^{m_{1}}\right) \cap r_{12}\left(\right.$ Bird $\left.^{m_{1}}\right)$, and $r_{12}\left(\right.$ Bird $\left.^{m_{1}}\right) \subseteq$ $r_{12}\left(\right.$ Fly $\left.^{m_{1}}\right)$. Therefore Penguin ${ }^{m_{2}} \subseteq r_{12}\left((\neg F l y)^{m_{1}}\right) \cap r_{12}\left(F^{\prime} l y^{m_{1}}\right) \subseteq \varnothing$ is not satisfiable.

An $\mathcal{E}$-connection-like constructed concept such as $\exists(i: E) .(j: D)$ can be defined in the module $i$, where $j: D$ is imported into $i$, with semantics: $\left\{x \in \Delta_{i} \mid \exists y \in\right.$ $\left.\left.\Delta_{j}, y^{\prime}=r_{j i}(y) \in \Delta_{i},\left(x, y^{\prime}\right) \in E^{m_{i}}, y \in D^{m_{j}}\right\}\right\} . \forall(i: E) .(j: D)$ can be constructed similarly. It is easy to see a combined model (Tableau) of $\mathcal{E}$-connections 77. can be reduced to a P-DL model by transforming every $\mathcal{E}$-connection instance $e(i: x, j: y)$ to a role instance $e\left(i: x, i: y^{\prime}\right)$ and adding $\left(y, y^{\prime}\right)$ to the domain relation $r_{j i}$ if it has not already been added.

Since "links" in $\mathcal{E}$-connections can be specified as local roles in P-DL with foreign concepts as ranges, link inclusion, link inverse, and link number restriction can also be reduced into normal role axioms in P-DL. Therefore, we have:

Theorem 5. $P-D L \mathcal{S H O I} \mathcal{Q P}_{\mathcal{C}}(D)$ is strictly more expressive than the $D D L$ extension to $\mathcal{S H O I} \mathcal{Q}$ with bridge rules between concepts, and $\mathcal{E}$-Connections $\mathcal{C}_{\mathcal{H} \mathcal{Q}}(\mathcal{S H I Q}, \mathcal{S H O Q}, \mathcal{S H I O})$ and $\mathcal{C}_{\mathcal{H}}^{\mathcal{E}}(\mathcal{S H I}, \mathcal{S H O Q}, \mathcal{S H I O})$.

Some types of DDL and $\mathcal{E}$-Connections can not be reduced to the P-DL extension $\mathcal{P}_{\mathcal{C}}$, e.g., DDL bridge rules between roles and individuals or generalized links [13] in $\mathcal{E}$-connections. However, we believe future extension of P-DL may cover some of these scenarios.

Another observation is that it is possible to simulate the one-to-one domain relations that are required in P-DL by the combination of DDL and Econnection: 2 . If we use bridge rules as a special type of $\mathcal{E}$-connections with " $\leq 1$ " cardinality restriction in $\mathcal{E}$-connections, it effectively encodes the one-toone domain relations. More precisely, for any pair of module $i, j$, if we denote $E$ as the $\mathcal{E}$-connection for bridge rules from $i$ to $j, F$ as the $\mathcal{E}$-connection for bridge rules from $j$ to $i$, , the following axioms can be added:

- In module $i$ : $\top_{i} \sqsubseteq \leq 1 E \cdot \top_{j}$

- In module $j: \top_{j} \sqsubseteq \leq 1 F . \top_{i}$

- $F=E^{-}$.

${ }^{2}$ We thank the anonymous reviewers of the Description Logics Workshop for pointing this out. 
However, such a simulation does not always meet the compositional consistency requirement of P-DL. Therefore, such a combination of DDL and $\mathcal{E}$ connections, while it can solve the inter-module unsatisfiability problem, may fail on some problems that require module transitive reusability, such as the general subsumption propagation problem as outlined in Example 1.

\section{Summary}

In this paper, we have investigated the semantics of DDL, $\mathcal{E}$-connections and PDL. We have shown that (a) one-to-one domain relation is a sufficient condition for exact DDL reasoning; (b) $\mathcal{E}$-connections, in general, are more expressive than DDL only with inverse links; c) an importing approach in P-DL can be used to ensure transitivity of inter-module subsumption without sacrificing the exactness of inference in P-DL with only a compromise of local semantics. Our results raise the possibility of avoiding many of the semantic difficulties in current modular ontology language proposals by removing the strong assumption of module disjointness.

Acknowledgements. This research was supported in part by grants from the US NSF (0219699, 0639230) and NIH (GM 066387).

\section{References}

1. F. Baader, C. Lutz, H. Sturm, and F. Wolter. Fusions of description logics. In Description Logics, pages 21-30, 2000.

2. J. Bao, D. Caragea, and V. Honavar. Modular ontologies - a formal investigation of semantics and expressivity. In R. Mizoguchi, Z. Shi, and F. Giunchiglia (Eds.): Asian Semantic Web Conference 2006, LNCS 4185, pages 616-631, 2006.

3. J. Bao, D. Caragea, and V. Honavar. Towards collaborative environments for ontology construction and sharing. In International Symposium on Collaborative Technologies and Systems (CTS 2006), pages 99-108. IEEE Press, 2006.

4. A. Borgida and L. Serafini. Distributed description logics: Directed domain correspondences in federated information sources. In CoopIS, pages 36-53, 2002.

5. P. Bouquet, F. Giunchiglia, and F. van Harmelen. C-OWL: Contextualizing ontologies. In Second International Semantic Web Conference, volume 2870 of Lecture Notes in Computer Science, pages 164-179. Springer Verlag, 2003.

6. C. Ghidini and L. Serafini. Frontiers Of Combining Systems 2, Studies in Logic and Computation, chapter Distributed First Order Logics, pages 121-140. Research Studies Press, 1998.

7. B. C. Grau. Combination and Integration of Ontologies on the Semantic Web. PhD thesis, Dpto. de Informatica, Universitat de Valencia, Spain, 2005.

8. B. C. Grau, B. Parsia, and E. Sirin. Tableau algorithms for e-connections of description logics. Technical report, University of Maryland Institute for Advanced Computer Studies (UMIACS), TR 2004-72, 2004.

9. B. C. Grau, B. Parsia, and E. Sirin. Working with multiple ontologies on the semantic web. In International Semantic Web Conference, pages 620-634, 2004. 
10. I. Horrocks and U. Sattler. A Tableaux Decision Procedure for SHOIQ. In IJCAI, pages 448-453, 2005.

11. O. Kutz, C. Lutz, F. Wolter, and M. Zakharyaschev. E-connections of description logics. In Description Logics Workshop, CEUR-WS Vol 81, 2003.

12. O. Kutz, C. Lutz, F. Wolter, and M. Zakharyaschev. E-connections of abstract description systems. Artif. Intell., 156(1):1-73, 2004.

13. B. Parsia and B. C. Grau. Generalized link properties for expressive epsilonconnections of description logics. In $A A A I$, pages 657-662, 2005.

14. L. Serafini, A. Borgida, and A. Tamilin. Aspects of distributed and modular ontology reasoning. In IJCAI, pages 570-575, 2005.

15. L. Serafini, H. Stuckenschmidt, and H. Wache. A formal investigation of mapping language for terminological knowledge. In IJCAI, pages 576-581, 2005.

16. L. Serafini and A. Tamilin. Drago: Distributed reasoning architecture for the semantic web. In ESWC, pages 361-376, 2005.

17. H. Stuckenschmidt, L. Serafini, and H. Wache. Reasoning about ontology mappings. Technical report, Department for Mathematics and Computer Science, University of Mannheim ; TR-2005-011, 2005. 